\title{
THE BISYMPLECTOMORPHISM GROUP OF A BOUNDED SYMMETRIC DOMAIN
}

\author{
ANTONIO J. DI SCALA, ANDREA LOI, AND GUY ROOS
}

\begin{abstract}
We determine the group of diffeomorphisms of a bounded symmetric domain, which preserve simultaneously the hyperbolic and the flat symplectic form.
\end{abstract}

\section{CONTEnTs}

Introduction

1. Hermitian positive Jordan triples

2. Symplectic forms in polar coordinates

3. Symplectic duality

4. The bisymplectomorphism group

References

\section{INTRODUCTION}

Let $\Omega$ be an Hermitian bounded symmetric domain in a complex vector space $V$; we always assume that $\Omega$ is given in its circled realization. The domain $\Omega$ is endowed with two natural symplectic forms: the flat form $\omega_{0}$ and the hyperbolic form $\omega_{-}$. In a similar way, the ambient vector space $V$ is also endowed with two natural symplectic forms: the Fubini-Study form $\omega_{+}$and the flat form $\omega_{0}$ (see Section 1 for the definition of $\left.\omega_{-}, \omega_{0}, \omega_{+}\right)$. It has been shown in [1] that there exists a diffeomorphism $F: \Omega \rightarrow V$ such that

$$
F^{*} \omega_{0}=\omega_{-}, \quad F^{*} \omega_{+}=\omega_{0} .
$$

This map is the same as the map $\psi$ which was used in 4, Theorem VII.4.3], with the property

$$
\psi^{*} \omega_{+}^{n}=\omega_{0}^{n}
$$

$\left(n=\operatorname{dim}_{\mathbb{C}} V\right)$, to show that the flat volume of a bounded symmetric domain, with some natural normalization, is equal to the degree of a canonical projective

Date: February 23, 2008.

2000 Mathematics Subject Classification. Primary 53D05, 58F06; Secondary 32M15, 17C10.

Key words and phrases. Kähler metrics; bounded symmetric domains; symplectic duality; Jordan triple systems; Bergman operator.

Research partially supported by GNSAGA (INdAM) and MIUR of Italy. 
embedding of its compact dual. In the one-dimensional case, where $V=\mathbb{C}$ and $\Omega$ is the unit disc $\Delta$, this map is simply $f: \Delta \rightarrow \mathbb{C}$ given by

$$
f(z)=\frac{z}{\sqrt{1-|z|^{2}}} \text {. }
$$

Even in this case, it does not seem that the property (0.1) had been noticed before. In the general case, the map $F$ may be defined by

$$
F(z)=B(z, z)^{-1 / 4} z,
$$

where $B(z, z)$ denotes the Bergman operator of the Jordan triple structure on $V$ associated to $\Omega$; it may also be defined by functional calculus in Hermitian positive Jordan triples. In view of the property (0.1), the map $F$ is called map of (bi)symplectic duality. The part $F^{*} \omega_{0}=\omega_{-}$tells that $F$ is a realization of the isomorphism of Mc Duff [3] for the bounded symmetric domain $\Omega$; but the property (0.1), which involves two pairs of symplectic forms, is much stronger. In order to determine all diffeomorphisms $F: \Omega \rightarrow V$ verifying (0.1), we determine the group of bisymplectomorphims of $\Omega$, that is, diffeomorphisms $\phi: \Omega \rightarrow \Omega$ such that

$$
\phi^{*} \omega_{0}=\omega_{0}, \quad \phi^{*} \omega_{-}=\omega_{-} .
$$

This group is infinite-dimensional, but is the direct product of the compact group $K$ of linear automorphisms of $\Omega$ with an infinite-dimensional Abelian group of "radial circular diffeomorphisms" (Theorem 4); this is the main result of this article and may be considered as a kind of Schwarz lemma.

The plan of this article is as follows. In Section 1 , we recall known facts about Jordan triple systems associated to bounded complex symmetric domains (see mainly [2]); the only result we could not find in the literature is Proposition 1, which describes the tangent space of the manifold of frames (the "Fürstenberg-Satake boundary" of $\Omega$ ) in terms of Peirce decomposition in Jordan triples. In Section 2. we compute the symplectic forms $\omega_{0}$ and $\omega_{\text {- }}$ using spectral decomposition in Jordan triples, which is the appropriate generalization of polar coordinates. From this, we derive in Section 3 a simple proof of the property (0.1), different from the proof given in 11. Section 4 is devoted to the study and characterization of bisymplectomorphisms.

\section{Hermitian POSITIVE JoRdan triples}

Let $\Omega$ be a bounded symmetric domain in a finite dimensional complex vector space $V$. We will always consider such a domain in its (unique up to linear isomorphism) circled realization. Consider the associated Jordan triple system $(V,\{,\}$,$) .$ For basic facts about Hermitian positive Jordan triples and their correspondence with complex symmetric domains, see [2, 4]. We recall hereunder those which will be used here.

1.1. Definitions and notations. Consider the operators on the Jordan triple $V$ defined by

$$
\begin{aligned}
D(x, y) z & =\{x, y, z\}, \\
Q(x, z) y & =\{x, y, z\}, \\
Q(x, x) & =2 Q(x), \\
B(x, y) & =\operatorname{id}_{V}-D(x, y)+Q(x) Q(y) .
\end{aligned}
$$


The operators $D(x, y)$ and $B(x, y)$ are $\mathbb{C}$-linear, the operator $Q(x)$ is $\mathbb{C}$-antilinear. The hermitian form

$$
(u \mid v)=\operatorname{tr} D(u, v)
$$

is a Hermitian scalar product on $V$; with respect to this product, $D(x, x)$ and $B(x, x)$ are self-adjoint.

For $z \in V$, the odd powers $z^{(2 p+1)}$ of $z$ in the Jordan triple system $V$ are defined by

$$
z^{(1)}=z, \quad z^{(2 p+1)}=Q(z) z^{(2 p-1)} .
$$

An element $e \in V$ is called tripotent if $e \neq 0$ and $e^{(3)}=e$. Two tripotents $e_{1}, e_{2}$ are called (strongly) orthogonal if $D\left(e_{1}, e_{2}\right)=0$. A tripotent element is called minimal, or primitive, if it is not the sum of two orthogonal tripotents. A tripotent element $e$ is called maximal if there is no tripotent orthogonal to $e$.

1.2. Spectral decomposition. Each element $z \in V$ has a unique spectral decomposition

$$
z=\lambda_{1} e_{1}+\cdots+\lambda_{s} e_{s} \quad\left(\lambda_{1}>\cdots>\lambda_{s}>0\right),
$$

where $\left(e_{1}, \ldots, e_{s}\right)$ is a sequence of pairwise orthogonal tripotents. The integer $s=\operatorname{rk} z$ is called the rank of $z$. Let $V_{z}^{+}$be the $\mathbb{R}$-subspace of $V$ generated by the odd powers $z, \ldots, z^{(2 p+1)}, \ldots$ and $V_{z}=V_{z}^{+} \oplus \mathrm{i} V_{z}^{+}$the $\mathbb{C}$-subspace generated by the odd powers of $z$. Then $\operatorname{rk} z=\operatorname{dim}_{\mathbb{R}} V_{z}^{+}$and $\left(e_{1}, \ldots, e_{s}\right)$ is an $\mathbb{R}$-basis of $V_{z}^{+}$. The rank of $V$ is $r=\operatorname{rk} V=\max \{\operatorname{rk} z \mid z \in V\}$; elements $z$ such that $\operatorname{rk} z=\operatorname{rk} V$ are called regular. If $z \in V$ is regular, with spectral decomposition

$$
z=\lambda_{1} e_{1}+\cdots+\lambda_{r} e_{r} \quad\left(\lambda_{1}>\cdots>\lambda_{r}>0\right),
$$

then $\left(e_{1}, \ldots, e_{r}\right)$ is a (Jordan) frame of $V$, that is, a maximal sequence of pairwise orthogonal minimal tripotents.

1.3. Peirce decompositions. Let $e \in V$ be a tripotent. Then the eigenvalues of $D(e, e)$ are contained in $\{0,1,2\}$. Define the Peirce subspaces of $e$ as

$$
V_{i}(e)=\{z \in V \mid D(e, e) z=i z\} \quad(i \in\{0,1,2\}) .
$$

The decomposition

$$
V=V_{0}(e) \oplus V_{1}(e) \oplus V_{2}(e)
$$

is called the Peirce decomposition of $V$ w.r. to $e$. A tripotent $e$ is maximal if $V_{0}(e)=0$, minimal if $V_{2}(e)=\mathbb{C} e$. The Peirce subspaces compose according to the law

$$
\left\{V_{i}(e), V_{j}(e), V_{k}(e)\right\} \subset V_{i-j+k}(e),
$$

where $V_{m}(e)=0$ if $m \notin\{0,1,2\}$; in particular, Peirce subspaces are Jordan subsystems of $V$. The $\mathbb{C}$-antilinear operator $Q(e)$ is 0 on $V_{0}(e) \oplus V_{1}(e)$; its restriction to $V_{2}(e)$ is involutive. Let

$$
V_{2}^{+}(e)=\{v \in V \mid D(e, e) v=2 v, Q(e) v=v\} .
$$

Then the decomposition of $V(e)$ into (real) eigenspaces of $Q(e)$ is

$$
V_{2}(e)=V_{2}^{+}(e) \oplus \mathrm{i} V_{2}^{+}(e) .
$$


Let $\mathbf{e}=\left(e_{1}, \ldots, e_{s}\right)$ be a sequence of pairwise orthogonal tripotents. Then the operators $D\left(e_{j}, e_{j}\right), 1 \leq j \leq s$ commute and have the common eigenspaces

$$
\begin{aligned}
& V_{j j}(\mathbf{e})=V_{2}\left(e_{j}\right) \quad(1 \leq j \leq s), \\
& V_{j k}(\mathbf{e})=V_{1}\left(e_{j}\right) \cap V_{1}\left(e_{k}\right) \quad(1 \leq j<k \leq s), \\
& V_{0 j}(\mathbf{e})=V_{1}\left(e_{j}\right) \cap \bigcap_{k \neq j} V_{0}\left(e_{k}\right) \quad(1 \leq j \leq s), \\
& V_{00}(\mathbf{e})=\bigcap_{k} V_{0}\left(e_{k}\right)
\end{aligned}
$$

(some of these subspaces may be 0 ). The decomposition

$$
V=\bigoplus_{0 \leq j \leq k \leq s} V_{j k}(\mathbf{e})
$$

is called the simultaneous Peirce decomposition of $V$ w.r. to e. If

$$
\begin{aligned}
& z=\lambda_{1} e_{1}+\cdots+\lambda_{s} e_{s} \quad\left(\lambda_{j} \in \mathbb{C}\right), \\
& e=e_{1}+\cdots+e_{s}
\end{aligned}
$$

and $v \in V_{j k}(\mathbf{e})$, then

$$
\begin{aligned}
D(z, z) v & =\left(\left|\lambda_{j}\right|^{2}+\left|\lambda_{k}\right|^{2}\right) v \\
Q(z) v & =\lambda_{j} \lambda_{k} Q(e) v \\
Q(z) Q(z) & =\left|\lambda_{j} \lambda_{k}\right|^{2} v, \\
B(z, z) v & =\left(1-\left|\lambda_{j}\right|^{2}\right)\left(1-\left|\lambda_{k}\right|^{2}\right) v, \\
B(z,-z) v & =\left(1+\left|\lambda_{j}\right|^{2}\right)\left(1+\left|\lambda_{k}\right|^{2}\right) v,
\end{aligned}
$$

where $\lambda_{0}=0$. So the $V_{j k}(\mathbf{e})$ 's are eigenspaces for all the operators $D(z, z), B(z, z)$, $B(z,-z), z=\lambda_{1} e_{1}+\cdots+\lambda_{s} e_{s}$.

1.4. Hermitian metrics and symplectic forms. Let $V$ be a Hermitian positive Jordan triple and let $\Omega$ be the associated Hermitian bounded symmetric domain. Let

$$
h_{0}(z)(u, v)=(u \mid v)=\operatorname{tr} D(u, v)
$$

be the flat Hermitian metric and let

$$
\begin{aligned}
\omega_{0}(z) & =\frac{\mathrm{i}}{2} \partial \bar{\partial}(z \mid z), \\
\omega_{0}(z)(u, v) & =\frac{\mathrm{i}}{2}((u \mid v)-(v \mid u))
\end{aligned}
$$

be the associated flat symplectic form. If $\Omega$ is endowed with the volume form $\omega_{0}^{n}$ $\left(n=\operatorname{dim}_{\mathbb{C}} V\right)$, the Bergman kernel of $\Omega$ is

$$
K(x, y)=\frac{C}{\operatorname{det} B(x, y)},
$$

with $C=\left(\int_{\Omega} \omega_{0}^{n}\right)^{-1}$. The Bergman metric of $\Omega$ is

$$
h_{-}(z)(u, v)=\partial_{u} \bar{\partial}_{v} \ln K(z, z)=-\partial_{u} \bar{\partial}_{v} \ln \operatorname{det} B(z, z) .
$$

It satisfies the relation

$$
h_{-}(z)(u, v)=h_{0}\left(B(z, z)^{-1} u, v\right) .
$$


In view of this relation, $B(z, z)$ is called the Bergman operator at $z \in \Omega$. by

The hyperbolic symplectic form of $\Omega$, associated to the Bergman metric, is defined

$$
\omega_{-}(z)=-\frac{i}{2} \partial \bar{\partial} \ln \operatorname{det} B(z, z) .
$$

From (1.22), it results that the forms $\omega_{0}$ and $\omega_{-}$are related with the Bergman operator by

$$
\omega_{-}(z)(u, v)=\omega_{0}\left(B(z, z)^{-1} u, v\right),
$$

for $z \in \Omega$ and $u, v \in T_{z} \Omega$.

The (generalized) Fubini-Study metric on $V$ is defined by

$$
h_{+}(z)(u, v)=\partial_{u} \bar{\partial}_{v} \ln \operatorname{det} B(z,-z) .
$$

The associated Kähler form is

$$
\omega_{+}(z)=\frac{\mathrm{i}}{2} \partial \bar{\partial} \ln \operatorname{det} B(z,-z) .
$$

It is related to the flat form by

$$
\omega_{+}(z)(u, v)=\omega_{0}\left(B(z,-z)^{-1} u, v\right) .
$$

1.5. Polar coordinates. Let $M$ be the set ot tripotents elements of the positive Jordan triple $V$. Then $M$ is a compact submanifold of $V$ (with connected components of different dimensions). At $e \in M$, the tangent space $T_{e} M$ and the normal space $N_{e} M$ to $M$ are

$$
\begin{aligned}
T_{e} M & =\mathrm{i} V_{2}^{+}(e) \oplus V_{1}(e), \\
N_{e} M & =V_{0}(e) \oplus V_{2}^{+}(e)
\end{aligned}
$$

(see [2, Theorem 5.6]).

The height $k$ of a tripotent element $e$ is the maximal length of a decomposition $e=e_{1}+\cdots+e_{k}$ into a sum of pairwise orthogonal (minimal) tripotents. Minimal tripotents have height 1 , maximal tripotents have height $r=\operatorname{rk} V$. Denote by $M_{k}$ the set of tripotents of height $k$. If $V$ is simple (that is, if $\Omega$ is irreducible), the submanifolds $M_{k}$ are the connected components of $M$.

The set $\mathcal{F}$ of frames (also called Fürstenberg-Satake boundary of $\Omega$ ):

$$
\mathcal{F}=\left\{\left(e_{1}, \ldots, e_{r}\right) \mid e_{j} \in M_{1}, e_{j} \perp e_{k}(1 \leq j<k \leq r)\right\},
$$

(where $e_{j} \perp e_{k}$ means orthogonality of tripotents: $D\left(e_{j}, e_{k}\right)=0$ or equivalently $\left.\left\{e_{j}, e_{j}, e_{k}\right\}=0\right)$ is a submanifold of $V^{r}$. If $\mathbf{e}=\left(e_{1}, \ldots, e_{r}\right)$ is a frame for a simple positive Hermitian Jordan triple $V$, the corresponding Peirce subspaces have the following properties (see [4, Theorem VI.3.5]):

- $V_{00}(\mathbf{e})=0$

- $V_{j j}(\mathbf{e})=\mathbb{C} e_{j}(1 \leq j \leq r)$

- all $V_{j k}(\mathbf{e})=V_{k j}(\mathbf{e})(1 \leq j<k \leq r)$ have the same dimension $a>0$;

- all $V_{0 j}(\mathbf{e})(1 \leq j \leq r)$ have the same dimension $b$; these subspaces are 0 if and only if the domain $\Omega$ is of "tube type".

The following proposition provides a description of the tangent space of $\mathcal{F}$. 
Proposition 1. Let $\mathbf{e}=\left(e_{1}, \ldots, e_{r}\right) \in \mathcal{F} \subset V^{r}$ and $e=e_{1}+\cdots+e_{r}$. Then $\left(v_{1}, \ldots, v_{r}\right) \in T_{\mathbf{e}} \mathcal{F}$ if and only if

$$
v_{j}=\mathrm{i} \alpha_{j} e_{j}+v_{j 0}+\sum_{\substack{1 \leq k \leq r \\ k \neq j}} v_{j k} \quad(1 \leq j \leq r),
$$

where $\alpha_{j} \in \mathbb{R}, v_{j 0} \in V_{0 j}(\mathbf{e}), v_{j k} \in V_{j k}(\mathbf{e})=V_{k j}(\mathbf{e})$ and

$$
Q(e) v_{j k}=-v_{k j} \quad(1 \leq j<k \leq r) .
$$

Proof. Let $\left(v_{1}, \ldots, v_{r}\right) \in T_{\mathbf{e}} \mathcal{F}$. As $e_{j}$ are minimal tripotents, we have

$$
v_{j} \in T_{e_{j}} M_{1}=\mathrm{i} \mathbb{R} e_{j} \oplus V_{1}\left(e_{j}\right)=\mathrm{i} \mathbb{R} e_{j} \oplus V_{0 j}(\mathbf{e}) \oplus \bigoplus_{\substack{1 \leq k \leq r \\ k \neq j}} V_{j k}(\mathbf{e}),
$$

which shows that $v_{j}$ has the form (1.29).

The orthogonality conditions in a frame are

$$
\left\{e_{j}, e_{j}, e_{k}\right\}=0 \quad(1 \leq j<k \leq r) .
$$

Differentiating these conditions yields

$$
\left\{v_{j}, e_{j}, e_{k}\right\}+\left\{e_{j}, v_{j}, e_{k}\right\}+\left\{e_{j}, e_{j}, v_{k}\right\}=0 \quad(1 \leq j<k \leq r) .
$$

As $D\left(e_{j}, e_{k}\right)=0$, this condition is reduced to

$$
Q\left(e_{j}, e_{k}\right) v_{j}+D\left(e_{j}, e_{j}\right) v_{k}=0 \quad(1 \leq j<k \leq r) .
$$

Let

$$
v_{j}=\mathrm{i} \alpha_{j} e_{j}+v_{j 0}+\sum_{\substack{1 \leq m \leq r \\ m \neq j}} v_{j m} \quad(1 \leq j \leq r) .
$$

Then

$$
\begin{aligned}
D\left(e_{j}, e_{j}\right) v_{k} & =v_{k j}, \quad Q\left(e_{j}, e_{k}\right) e_{j}=0, \\
Q\left(e_{j}, e_{k}\right) & =Q\left(e_{j}+e_{k}\right)-Q\left(e_{j}\right)-Q\left(e_{k}\right),
\end{aligned}
$$

and we get from (1.17)

$$
\begin{aligned}
Q\left(e_{j}+e_{k}\right) v_{j m} & =\delta_{k}^{m} Q(e) v_{j m}, \quad Q\left(e_{j}\right) v_{j m}=0, \quad Q\left(e_{k}\right) v_{j m}=0, \\
Q\left(e_{j}, e_{k}\right) v_{j} & =Q(e) v_{j k} .
\end{aligned}
$$

This shows that the conditions (1.31) are equivalent to (1.30).

Comparing the description of $T_{\mathbf{e}} \mathcal{F}$ with the simultaneous Peirce decomposition of $V$ w.r. to e, it is easily checked that $T_{\mathbf{e}} \mathcal{F}$ is a real vector space of dimension $2 n-r$, where $n=\operatorname{dim}_{\mathbb{C}} V$. This implies that the map

$$
\begin{aligned}
\left\{\lambda_{1}>\cdots>\lambda_{r}>0\right\} \times \mathcal{F} & \rightarrow V_{\text {reg }} \\
\left(\left(\lambda_{1}, \ldots, \lambda_{r}\right),\left(e_{1}, \ldots, e_{r}\right)\right) & \mapsto \sum \lambda_{j} e_{j}
\end{aligned}
$$

is a diffeomorphism onto the set $V_{\text {reg }}$ of regular elements of $V$; its restriction

$$
\left\{1>\lambda_{1}>\cdots>\lambda_{r}>0\right\} \times \mathcal{F} \rightarrow \Omega_{\text {reg }}
$$

is a diffeomorphism onto the set $\Omega_{\text {reg }}$ of regular elements of $\Omega$. This map plays the same role as polar coordinates in rank one. 
1.6. Functional calculus. See [2, Section 3.18]. Using the spectral decomposition, it is possible to associate to an odd function $f:(-1,1) \rightarrow \mathbb{C}$ (resp. $f: \mathbb{R} \rightarrow \mathbb{C}$ ) a "radial" map $F: \Omega \rightarrow V$ (resp. $F: V \rightarrow V$ ) in the following way. Let $z \in V$ and let

$$
z=\lambda_{1} e_{1}+\cdots+\lambda_{k} e_{k}, \quad \lambda_{1}>\cdots>\lambda_{k}>0
$$

be the spectral decomposition of $z$. Define the map $F=\tilde{f}$ associated to $f$ by

$$
F(z)=f\left(\lambda_{1}\right) e_{1}+\cdots+f\left(\lambda_{k}\right) e_{k} .
$$

Since $f$ is odd, it follows from properties of tripotents that, for

$$
z=\lambda_{1} e_{1}+\cdots+\lambda_{r} e_{r}
$$

where $\mathbf{e}=\left(e_{1}, \ldots, e_{r}\right)$ is a frame and $\lambda_{1}, \ldots, \lambda_{r} \in \mathbb{R}$, we have

$$
F(z)=f\left(\lambda_{1}\right) e_{1}+\cdots+f\left(\lambda_{r}\right) e_{r} .
$$

The linear correspondence $f \mapsto F$ has the following properties:

(1) If $f$ is continuous, then $F$ is continuous.

(2) If

$$
f(t)=\sum_{k=0}^{N} a_{k} t^{2 k+1}
$$

is a polynomial, then $F$ is the map defined by

$$
F(z)=\sum_{k=0}^{N} a_{k} z^{(2 k+1)} \quad(z \in V) .
$$

(3) If $f$ is analytic, then $F$ is real-analytic; if $f$ is given near 0 by

$$
f(t)=\sum_{k=0}^{\infty} a_{k} t^{2 k+1}
$$

then $F$ has the Taylor expansion near $0 \in V$ :

$$
F(z)=\sum_{k=0}^{\infty} a_{k} z^{(2 k+1)} .
$$

(4) If $|f(\lambda)| \leq C|\lambda|^{2 N+1}$ for $|\lambda|<c$, then $\|F(z)\| \leq C\|z\|^{2 N+1}$ for $z \in V$, $\|z\|<c$. (Here $\|z\|$ denotes the spectral norm of $z$ in $V:\|z\|=\max \left|\lambda_{j}\right|$ for $z=\lambda_{1} e_{1}+\cdots+\lambda_{r} e_{r}$, where $\mathbf{e}=\left(e_{1}, \ldots, e_{r}\right)$ is a frame $)$.

(5) If $f$ is $C^{\infty}$, then $F$ is also $C^{\infty}$.

Properties (1)-(4) are easy. Property (5) follows from (2) and (4).

\section{SyMPLECTIC FORMS IN POLAR COORDiNATES}

2.1. The flat symplectic form. We compute the flat symplectic form $\omega_{0}$ in the "polar coordinates"

$$
\begin{aligned}
\left\{\lambda_{1}>\cdots>\lambda_{r}>0\right\} \times \mathcal{F} & \rightarrow V_{\text {reg }} \\
\left(\left(\lambda_{1}, \ldots, \lambda_{r}\right),\left(e_{1}, \ldots, e_{r}\right)\right) & \mapsto \sum \lambda_{j} e_{j} .
\end{aligned}
$$


If $\left(z^{1}, \ldots, z^{n}\right)(n=\operatorname{dim} V)$ are orthonormal coordinates for the Hermitian product $(u \mid v)$, then

$$
\omega_{0}=\frac{i}{2} \sum_{m=1}^{n} \mathrm{~d} z^{m} \wedge \mathrm{d} \bar{z}^{m}
$$

Let $\left(e_{j}^{m}\right)_{1 \leq m \leq n}$ be the coordinates of $e_{j}$ in the same basis as for $z$. From

$$
z=\sum_{j=1}^{r} \lambda_{j} e_{j}, \quad z^{m}=\sum_{j=1}^{r} \lambda_{j} e_{j}^{m}
$$

we have

$$
\begin{aligned}
\mathrm{d} z^{m} \wedge \mathrm{d} \bar{z}^{m}= & \sum_{j, k=1}^{r} \lambda_{j} \lambda_{k} \mathrm{~d} e_{j}^{m} \wedge \mathrm{d} \bar{e}_{k}^{m}+\sum_{j, k=1}^{r} e_{j}^{m} \bar{e}_{k}^{m} \mathrm{~d} \lambda_{j} \wedge \mathrm{d} \lambda_{k} \\
& +\sum_{j, k=1}^{r} \lambda_{k} \mathrm{~d} \lambda_{j} \wedge\left(e_{j}^{m} \mathrm{~d} \bar{e}_{k}^{m}-\bar{e}_{k}^{m} \mathrm{~d} e_{j}^{m}\right) .
\end{aligned}
$$

Using $\left(e_{j} \mid e_{k}\right)=\delta_{j k}$, we get

$$
\omega_{0}=\sum_{j, k=1}^{r} \lambda_{j} \lambda_{k} \omega_{j k}+\sum_{j, k=1}^{r} \lambda_{k} \mathrm{~d} \lambda_{j} \wedge \eta_{j k}
$$

where

$$
\begin{aligned}
& \eta_{j k}=\left.\frac{\mathrm{i}}{2} \sum_{m=1}^{n} e_{j}^{m} \mathrm{~d} \bar{e}_{k}^{m}\right|_{\mathcal{F}}, \\
& \omega_{j k}=\left.\frac{\mathrm{i}}{2} \sum_{m=1}^{n} \mathrm{~d} e_{j}^{m} \wedge \mathrm{d} \bar{e}_{k}^{m}\right|_{\mathcal{F}}=\mathrm{d} \eta_{j k} .
\end{aligned}
$$

We compute $\eta_{j k}$ and $\omega_{j k}$ using the description of $T_{\mathbf{e}} \mathcal{F}$ given in Proposition 1 Let $v, w \in T_{\mathbf{e}} \mathcal{F}$ with $v=\left(v_{1}, \ldots, v_{r}\right), w=\left(w_{1}, \ldots, w_{r}\right)$,

$$
\begin{array}{ll}
v_{j}=\mathrm{i} \alpha_{j} e_{j}+v_{j 0}+\sum_{\substack{1 \leq m \leq r \\
m \neq j}} v_{j m} \quad(1 \leq j \leq r), \\
w_{j}=\mathrm{i} \beta_{j} e_{j}+w_{j 0}+\sum_{\substack{1 \leq m \leq r \\
m \neq j}} w_{j m} \quad(1 \leq j \leq r),
\end{array}
$$

where

$$
\begin{aligned}
\alpha_{j}, \beta_{j} & \in \mathbb{R}, \quad v_{j 0}, w_{j 0} \in V_{0 j}(\mathbf{e}), \quad v_{j k}, w_{j k} \in V_{j k}(\mathbf{e}), \\
Q(e) v_{j k} & =-v_{k j}, \quad Q(e) w_{j k}=-w_{k j} \quad(1 \leq j<k \leq r) .
\end{aligned}
$$

Then, as the Peirce subspaces are orthogonal w.r. to ( $\mid$ ), we deduce from (2.2)-(2.3)

$$
\begin{aligned}
\eta_{j j}(\mathbf{e})(v) & =\frac{\mathrm{i}}{2}\left(e_{j} \mid v_{j}\right)=\frac{1}{2} \alpha_{j}, \\
\eta_{j k}(\mathbf{e})(v) & =\frac{\mathrm{i}}{2}\left(e_{j} \mid v_{k}\right)=0 \quad(j \neq k), \\
\omega_{j k} & =\mathrm{d} \eta_{j k}=0 \quad(j \neq k)
\end{aligned}
$$

and

$$
\omega_{j j}(\mathbf{e})(v, w)=\frac{\mathrm{i}}{2}\left(\left(v_{j} \mid w_{j}\right)-\left(w_{j} \mid v_{j}\right)\right)
$$




$$
=\left\langle v_{j 0} \mid w_{j 0}\right\rangle+\sum_{\substack{1 \leq m \leq r \\ m \neq j}}\left\langle v_{j m} \mid w_{j m}\right\rangle,
$$

where $\langle\mid\rangle$ denotes the symplectic product

$$
\langle x \mid y\rangle=\frac{\mathrm{i}}{2}((x \mid y)-(y \mid x)) .
$$

Finally, we have

$$
\omega_{0}=\sum_{j=1}^{r} \lambda_{j}^{2} \omega_{j j}+\sum_{j=1}^{r} \lambda_{j} \mathrm{~d} \lambda_{j} \wedge \eta_{j j}
$$

with $\omega_{j j}$ and $\eta_{j j}$ given by (2.5), (2.4). The expression (2.5) shows that the $\omega_{j j}$ 's $(1 \leq j \leq r)$ are linearly independent at each point $\mathbf{e} \in \mathcal{F}$.

As

$$
\begin{aligned}
2(Q(z) x \mid y) & =(D(z, x) z \mid y)=(z \mid D(x, z) y) \\
& =(z \mid D(y, z) x)=(D(z, y) z \mid x)=2(Q(z) y \mid x)
\end{aligned}
$$

the $Q$ operator satisfies

$$
\begin{aligned}
& (Q(z) x \mid y)=(Q(z) y \mid x), \\
& \langle Q(z) x \mid y\rangle=-\langle x \mid Q(z) y\rangle
\end{aligned}
$$

for all $z, x, y \in V$.

For $v, w \in T_{\mathbf{e}} \mathcal{F}$, using

$$
Q(e) v_{j k}=-v_{k j}, \quad Q(e) w_{j k}=-w_{k j} \quad(1 \leq j \neq k \leq r),
$$

we have then

$$
\left\langle v_{j k} \mid w_{j k}\right\rangle=\left\langle Q(e) v_{k j} \mid Q(e) w_{k j}\right\rangle=-\left\langle v_{k j} \mid Q(e)^{2} w_{k j}\right\rangle,
$$

that is,

$$
\left\langle v_{j k} \mid w_{j k}\right\rangle=-\left\langle v_{k j} \mid w_{k j}\right\rangle,
$$

as $w_{k j} \in V_{2}(e)$ and $Q(e)$ is involutive on $V_{2}(e)$. In view of (2.10), the flat symplectic form $\omega_{0}$ in polar coordinates may be rewritten

$$
\omega_{0}=\sum_{j=1}^{r} \lambda_{j}^{2} \theta_{j 0}+\sum_{\substack{j, k \\ 1 \leq j<k \leq r}}\left(\lambda_{j}^{2}-\lambda_{k}^{2}\right) \theta_{j k}+\sum_{j=1}^{r} \lambda_{j} \mathrm{~d} \lambda_{j} \wedge \eta_{j j}
$$

where the $\eta_{j j}$ 's are defined by (2.4), and the $\theta_{j 0}$ 's and $\theta_{j k}$ 's by

$$
\begin{array}{ll}
\theta_{j 0}(\mathbf{e})(v, w)=\left\langle v_{j 0} \mid w_{j 0}\right\rangle, & (1 \leq j \leq r), \\
\theta_{j k}(\mathbf{e})(v, w)=\left\langle v_{j k} \mid w_{j k}\right\rangle & (1 \leq j<k \leq r) .
\end{array}
$$

for $v, w \in T_{\mathbf{e}} \mathcal{F}$. Note that these forms are (pull-backs of) forms on the manifold of frames $\mathcal{F}$, and that the Peirce subspaces $V_{0 j}(\mathbf{e})$, hence the $\theta_{j 0}$ 's, are 0 when the domain is of tube type. 
2.2. Hyperbolic form and Fubini-Study form. We compute now the hyperbolic form $\omega_{-}$and the Fubini-Study form $\omega_{+}$in polar coordinates.

Using

$$
\begin{aligned}
B(z, z)^{-1} v_{j 0} & =\left(1-\lambda_{j}^{2}\right)^{-1} v_{j 0} \quad(1 \leq j \leq r), \\
B(z, z)^{-1} v_{j k} & =\left(1-\lambda_{j}^{2}\right)^{-1}\left(1-\lambda_{k}^{2}\right)^{-1} v_{j k} \quad(1 \leq j<k \leq r), \\
B(z, z)^{-1} e_{j} & =\left(1-\lambda_{j}^{2}\right)^{-2} e_{j} \quad(1 \leq j \leq r)
\end{aligned}
$$

in (2.11), (2.12), (2.13), we obtain

$$
\begin{aligned}
\eta_{j j}(\mathbf{e})\left(B(z, z)^{-1} v\right) & =\left(1-\lambda_{j}^{2}\right)^{-2} \eta_{j j}(\mathbf{e})(v), \\
\theta_{j 0}(\mathbf{e})\left(B(z, z)^{-1} v, w\right) & =\left(1-\lambda_{j}^{2}\right)^{-1} \theta_{j 0}(\mathbf{e})(v, w), \\
\theta_{j k}(\mathbf{e})\left(B(z, z)^{-1} v, w\right) & =\left(1-\lambda_{j}^{2}\right)^{-1}\left(1-\lambda_{k}^{2}\right)^{-1} \theta_{j k}(\mathbf{e})(v, w),
\end{aligned}
$$

for $z=\lambda_{1} e_{1}+\cdots+\lambda_{r} e_{r}$ and $v, w \in T_{\mathbf{e}} \mathcal{F}$. From (1.24):

$$
\omega_{-}(z)(v, w)=\omega_{0}\left(B(z, z)^{-1} v, w\right)
$$

and the expression of $\omega_{0}$ in polar coordinates, we have

$$
\omega_{-}=\sum_{j=1}^{r} \frac{\lambda_{j}^{2}}{1-\lambda_{j}^{2}} \theta_{j 0}+\sum_{\substack{j, k \\ 1 \leq j<k \leq r}} \frac{\lambda_{j}^{2}-\lambda_{k}^{2}}{\left(1-\lambda_{j}^{2}\right)\left(1-\lambda_{k}^{2}\right)} \theta_{j k}+\sum_{j=1}^{r} \frac{\lambda_{j} \mathrm{~d} \lambda_{j}}{\left(1-\lambda_{j}^{2}\right)^{2}} \wedge \eta_{j j} .
$$

In the same way, the Fubini-Study symplectic form on $V$ is

$$
\omega_{+}=\sum_{j=1}^{r} \frac{\lambda_{j}^{2}}{1+\lambda_{j}^{2}} \theta_{j 0}+\sum_{\substack{j, k \\ 1 \leq j<k \leq r}} \frac{\lambda_{j}^{2}+\lambda_{k}^{2}}{\left(1+\lambda_{j}^{2}\right)\left(1+\lambda_{k}^{2}\right)} \theta_{j k}+\sum_{j=1}^{r} \frac{\lambda_{j} \mathrm{~d} \lambda_{j}}{\left(1+\lambda_{j}^{2}\right)^{2}} \wedge \eta_{j j} .
$$

\section{Symplectic DuAlity}

Consider the real analytic maps $f=]-1,1[\rightarrow \mathbb{R}$ and $g: \mathbb{R} \rightarrow]-1,1[$, inverse of each other, defined by

$$
\begin{array}{ll}
f(t)=\frac{t}{\sqrt{1-t^{2}}} & (-1<t<1), \\
g(t)=\frac{t}{\sqrt{1+t^{2}}} \quad(t \in \mathbb{R}) .
\end{array}
$$

By the functional calculus described in Subsection 1.6, we associate to these maps the real analytic diffeomorphisms, also inverse of each other

$$
\begin{aligned}
& F=\widehat{f}: \Omega \rightarrow V, \\
& G=\widehat{g}: V \rightarrow \Omega,
\end{aligned}
$$

where $\Omega$ is the bounded symmetric domain associated to the Jordan triple $V$. If $\mathbf{e}=\left(e_{1}, \ldots, e_{r}\right)$ is a frame and $z=\sum_{j=1}^{r} \lambda_{j} e_{j}$, then

$$
F(z)=\sum_{j=1}^{r} \frac{\lambda_{j}}{\sqrt{1-\lambda_{j}^{2}}} e_{j} \quad(z \in \Omega)
$$




$$
G(z)=\sum_{j=1}^{r} \frac{\lambda_{j}}{\sqrt{1+\lambda_{j}^{2}}} e_{j} \quad(z \in V) .
$$

Using (1.16)-(1.20), the maps $F$ and $G$ may also be defined by

$$
\begin{aligned}
& F(z)=B(z, z)^{-1 / 4} z=\left(\operatorname{id}_{V}-\frac{1}{2} D(z, z)\right)^{-1 / 2} z \quad(z \in \Omega), \\
& G(z)=B(z,-z)^{-1 / 4} z=\left(\operatorname{id}_{V}-\frac{1}{2} D(z,-z)\right)^{-1 / 2} z \quad(z \in V) .
\end{aligned}
$$

The following theorem is the main result of [1]. We give here a different and simpler proof, using the expression of the symplectic forms $\omega_{0}, \omega_{-}, \omega_{+}$in generalized polar coordinates.

Theorem 1. (Symplectic duality)

$$
\begin{array}{ll}
F^{*} \omega_{0}=\omega_{-}, & F^{*} \omega_{+}=\omega_{0}, \\
G^{*} \omega_{0}=\omega_{+}, & G^{*} \omega_{-}=\omega_{0} .
\end{array}
$$

Proof. In polar coordinates, the map $F$ is written

$$
\left(\left(\lambda_{1}, \ldots, \lambda_{r}\right), \mathbf{e}\right) \mapsto\left(\left(\mu_{1}, \ldots, \mu_{r}\right), \mathbf{e}\right)
$$

with

$$
\mu_{j}=\frac{\lambda_{j}}{\sqrt{1-\lambda_{j}^{2}}}
$$

As, by (2.11),

$$
\omega_{0}=\sum_{j=1}^{r} \lambda_{j}^{2} \theta_{j 0}+\sum_{\substack{j, k \\ 1 \leq j<k \leq r}}\left(\lambda_{j}^{2}-\lambda_{k}^{2}\right) \theta_{j k}+\sum_{j=1}^{r} \lambda_{j} \mathrm{~d} \lambda_{j} \wedge \eta_{j j},
$$

we obtain, using (3.9),

$$
\begin{aligned}
F^{*} \omega_{0}= & \sum_{j=1}^{r} \frac{\lambda_{j}^{2}}{1-\lambda_{j}^{2}} \theta_{j 0}+\sum_{\substack{j, k \\
1 \leq j<k \leq r}}\left(\frac{\lambda_{j}^{2}}{1-\lambda_{j}^{2}}-\frac{\lambda_{k}^{2}}{1-\lambda_{k}^{2}}\right) \theta_{j k} \\
& +\sum_{j=1}^{r} \frac{\lambda_{j} \mathrm{~d} \lambda_{j}}{\left(1-\lambda_{j}^{2}\right)^{2}} \wedge \eta_{j j},
\end{aligned}
$$

which, compared to (2.17), gives $F^{*} \omega_{0}=\omega_{-}$on the open dense subset $\Omega_{\text {reg }}$ of regular elements, and by continuity on all of $\Omega$.

The relation $G^{*} \omega_{0}=\omega_{+}$is proved along the same lines. The relations $F^{*} \omega_{+}=\omega_{0}$ and $G^{*} \omega_{-}=\omega_{0}$ follow, as $F$ and $G$ are inverse of each other.

In view of this theorem, the map $F$ (or the map $G=F^{-1}$ ) is called the duality map.

Example 1. (Type $\left.I_{1,1}\right)$ Here $V=\mathbb{C}, \Omega$ is the unit disc,

$$
\omega_{0}=\frac{\mathrm{i}}{2} \mathrm{~d} z \wedge \mathrm{d} \bar{z}, \quad \omega_{-}=\frac{\mathrm{i}}{2} \frac{\mathrm{d} z \wedge \mathrm{d} \bar{z}}{(1-z \bar{z})^{2}}, \quad \omega_{+}=\frac{\mathrm{i}}{2} \frac{\mathrm{d} z \wedge \mathrm{d} \bar{z}}{(1+z \bar{z})^{2}} .
$$

The duality map is

$$
F(z)=\frac{z}{\sqrt{1-z \bar{z}}} .
$$


Example 2. (Type $\left.I_{1, n}\right)$ Here $V=\mathbb{C}^{n}$ with the Hermitian norm $\|z\|^{2}=\sum z_{j} \bar{z}_{j}, \Omega$ is the unit Hermitian ball,

$$
\omega_{0}=\frac{\mathrm{i}}{2} \sum \mathrm{d} z_{j} \wedge \mathrm{d} \bar{z}_{j}, \quad \omega_{-}=\frac{\omega_{0}}{\left(1-\|z\|^{2}\right)^{n+1}}, \quad \omega_{+}=\frac{\omega_{0}}{\left(1+\|z\|^{2}\right)^{n+1}} .
$$

The duality map is

$$
F(z)=\frac{z}{\sqrt{1-\|z\|^{2}}} .
$$

\section{The BISYMPLECTOMORPHISM GROUP}

4.1. In this section we study to which extent a diffeomorphism $F: \Omega \rightarrow V$ satisfying the property (3.7) is unique. If two diffeomorphisms $F_{1}, F_{2}: \Omega \rightarrow V$ satisfy (3.7), then $F_{2}=F_{1} \circ f$, where $f: \Omega \rightarrow \Omega$ preserves $\omega_{0}$ and $\omega_{-}$. This leads us to the following definition.

Definition 1. A bisymplectomorphism of $\Omega$ is a diffeomorphism $f: \Omega \rightarrow \Omega$ which satisfies

$$
\begin{aligned}
f^{*} \omega_{0} & =\omega_{0}, \\
f^{*} \omega_{-} & =\omega_{-} .
\end{aligned}
$$

Clearly, bisymplectomorphisms of $\Omega$ form a group, which will be denoted by $\mathcal{B}(\Omega)$ and called the bisymplectomorphism group of $\Omega$.

From (1.24), we derive a characterization of $\mathcal{B}(\Omega)$ in terms of the Bergman operator:

Proposition 2. Let $\Omega$ be a bounded symmetric domain. Then a diffeomorphism $f \in \operatorname{Diff}(\Omega)$ is a bisymplectomorphism if and only if it satisfies

$$
\begin{aligned}
f^{*} \omega_{0} & =\omega_{0}, \\
B(f(z), f(z)) \circ \mathrm{d} f(z) & =\mathrm{d} f(z) \circ B(z, z) \quad(z \in \Omega) .
\end{aligned}
$$

Note that the second condition implies that the tangent map d $f(z)$ maps invariant subspaces of $B_{z}=B(z, z)$ to invariant subspaces of $B_{f(z)}$, and that $B_{f(z)}$ has the same eigenvalues as $B_{z}$.

Proof. Let $z \in \Omega, u, v \in T_{z} \Omega$. We have from (1.24) and (4.1)

$$
\begin{aligned}
\omega_{-}(z)(u, v) & =\omega_{0}\left(B(z, z)^{-1} u, v\right) \\
& =\omega_{0}\left(\mathrm{~d} f(z) B(z, z)^{-1} u, \mathrm{~d} f(z) v\right), \\
\left(f^{*} \omega_{-}\right)(z)(u, v) & =\omega_{-}(f(z))(\mathrm{d} f(z) u, \mathrm{~d} f(z) v) \\
& =\omega_{0}\left(B(f(z), f(z))^{-1} \mathrm{~d} f(z) u, \mathrm{~d} f(z) v\right),
\end{aligned}
$$

so that, assuming (4.1), the condition (4.2) is equivalent to

$$
\omega_{0}\left(\mathrm{~d} f(z) B(z, z)^{-1} u, \mathrm{~d} f(z) v\right)=\omega_{0}\left(B(f(z), f(z))^{-1} \mathrm{~d} f(z) u, \mathrm{~d} f(z) v\right)
$$

for all $u, v$. As $\omega_{0}$ is non singular and $\mathrm{d} f(z)$ is bijective, this is equivalent to

$$
\mathrm{d} f(z) \circ B(z, z)^{-1}=B(f(z), f(z))^{-1} \circ \mathrm{d} f(z),
$$

that is, to (4.4). 
4.2. Here we study diffeomorphisms of $\Omega$ satisfying the condition (4.4). Recall that $B_{z}=B(z, z): V \rightarrow V$ is a $\mathbb{C}$-linear operator, self-adjoint w.r. to the Hermitian metric $h_{0}$, positive if $z \in \Omega$. Let $r$ denote the rank of $\Omega$ and $V$.

For $z \in \Omega$, consider the spectral decomposition

$$
z=\lambda_{1} e_{1}+\lambda_{2} e_{2}+\cdots+\lambda_{s} e_{s}, \quad 1>\lambda_{1}>\lambda_{2}>\cdots>\lambda_{s}>0
$$

where $s=\operatorname{rk} z \leq r=\operatorname{rk} V$. An element is called regular if $\operatorname{rk} z=\operatorname{rk} V$; for regular elements, which form an open dense subset of $\Omega$, the decomposition (4.5) is the decomposition using generalized polar coordinates.

Let

$$
V=\bigoplus_{0 \leq i \leq j \leq s} V_{i j}
$$

be the simultaneous Peirce decomposition relative to $\left(e_{1}, \ldots, e_{s}\right)$. Note that some subspaces $V_{i j}$ may be 0 . The operator $B(z, z)$ may only have the eigenvalues

$$
\begin{array}{rll}
\left(1-\lambda_{i}^{2}\right)^{2} & (1 \leq i \leq s), \quad\left(1-\lambda_{i}^{2}\right)\left(1-\lambda_{j}^{2}\right) \quad(1 \leq i<j \leq s), \\
\left(1-\lambda_{i}^{2}\right) & (1 \leq i \leq s), \quad 1,
\end{array}
$$

which occur respectively on the subspaces

$$
V_{i i}, \quad V_{i j}, \quad V_{0 i}, \quad V_{00} .
$$

The relation (4.4) then implies that $B(z, z)$ and $B(f(z), f(z))$ have the same eigenvalues with the same multiplicities. Moreover, if all eigenvalues in the list (4.6) occurring for non-zero $V_{i j}$ are different, the non-zero subspaces of the list (4.7) are the eigenspaces of $B(z, z)$ and are mapped by $\mathrm{d} f(z)$ to the corresponding eigenspaces of $B(f(z), f(z))$.

Definition 2. Let $\Omega$ be an irreducible bounded symmetric domain and denote by $(V,\{,\}$,$) be the corresponding Jordan triple. An element z \in V$ is called superregular if $z$ is regular and if all eigenvalues in the list (4.6), occurring on non-zero $V_{i j}$, are different.

If $V$ is of tube type or of rank 1 , any regular element $z \in V$ is super-regular. If $V$ is not of tube type, an element $z \in V$ is super-regular if it is regular and if its spectral values satisfy

$$
1-\lambda_{i}^{2} \neq\left(1-\lambda_{j}^{2}\right)^{2}
$$

for all $(i, j), i<j$. Clearly, super-regular elements form an open dense subset of $V$.

Let $z \in \Omega$ be an element of rank one. Then the spectral decomposition of $z$ is

$$
z=\lambda_{1} e_{1}, \quad 0<\lambda_{1}<1,
$$

where $e_{1}$ is a tripotent; the associated Peirce decomposition is

$$
V_{11}=V_{2}\left(e_{1}\right), \quad V_{01}=V_{1}\left(e_{1}\right), \quad V_{00}=V_{0}\left(e_{1}\right)
$$

and the eigenvalues of $B(z, z)$ on these subspaces are respectively

$$
\left(1-\lambda_{1}^{2}\right)^{2}, \quad 1-\lambda_{1}^{2}, \quad 1 .
$$

It then follows that

$$
f(z)=\lambda_{1} \varepsilon_{1},
$$


where $\varepsilon_{1}$ is a tripotent such that $V_{2}\left(\varepsilon_{1}\right)=\mathrm{d} f(z) V_{2}\left(e_{1}\right)$, which means that $e_{1}$ and $\varepsilon_{1}$ have the same height $\operatorname{dim} V_{2}\left(e_{1}\right)=\operatorname{dim} V_{2}\left(\varepsilon_{1}\right)$. In particular, if $e_{1}$ is minimal (resp. maximal), then $\varepsilon_{1}$ is minimal (resp. maximal).

Let $V_{z}$ be the $\mathbb{C}$-subspace generated by the odd powers $z, \ldots, z^{(2 p+1)}, \ldots$ Then $\operatorname{dim}_{\mathbb{C}} V_{z}=\operatorname{rk} z \leq r$, and $\operatorname{dim}_{\mathbb{C}} V_{z}=r$ if and only if $z$ is a regular element. Denote

$$
P_{z}=V_{z} \cap \Omega \text {. }
$$

For $z \in \Omega, z \neq 0$ with spectral decomposition $z=\sum_{j=1}^{k} \alpha_{j} e_{j}\left(\alpha_{1}>\cdots>\alpha_{k}>0\right.$; $\left.k=\operatorname{rk}_{V} x\right), P_{z}$ is the $k$-polydisc

$$
P_{z}=\left\{u=\sum u_{j} e_{j}|| u_{j} \mid<1\right\} .
$$

Note that if $u \in P_{z}$ and $\operatorname{rk}_{V} u=\operatorname{rk}_{V} z$, then $V_{u}=V_{z}$ and $P_{u}=P_{z}$.

Lemma 3. Let $f: \Omega \rightarrow \Omega$ be a diffeomorphism of $\Omega$ such that

$$
B(f(z), f(z)) \circ \mathrm{d} f(z)=\mathrm{d} f(z) \circ B(z, z)
$$

for all $z \in \Omega$. Then for all $z \in \Omega$,

$$
\mathrm{d} f(z) V_{z}=V_{f(z)} \text {. }
$$

Proof. Let $z$ be a super-regular element and let

$$
z=\alpha_{1} e_{1}+\cdots+\alpha_{r} e_{r}
$$

be the spectral decomposition of $z$ in $V$; then $V_{z}:=\mathbb{C} e_{1} \oplus \cdots \oplus \mathbb{C} e_{r}$ is the sum of the eigenspaces of $B(z, z)$ relative to the eigenvalues

$$
\left(1-\alpha_{j}^{2}\right)^{2} \quad(1 \leq j \leq r)
$$

so that it follows from (4.4) that

$$
\mathrm{d} f(z) V_{z}=V_{f(z)} \text {. }
$$

By continuity, (4.9) holds for all $z \in \Omega$.

Proposition 4. Let $f: \Omega \rightarrow \Omega$ be a diffeomorphism of $\Omega$ such that

$$
B(f(z), f(z)) \circ \mathrm{d} f(z)=\mathrm{d} f(z) \circ B(z, z)
$$

for all $z \in \Omega$. Then for any element $z \in \Omega, z \neq 0$, we have

$$
f\left(P_{z}\right)=P_{f(z)} \text {. }
$$

Proof. Let $s=\operatorname{rk}_{V} z$. We already know that $\mathrm{d} f(z) V_{z}=V_{f(z)}$, which implies that $\operatorname{rk}_{V} f(z)=\operatorname{rk}_{V} z$. So there exist continuous functions $\beta_{j}$ such that

$$
\mathrm{d} f(z) u=\sum_{j=1}^{s} \beta_{j}(u, z)(f(z))^{(2 j+1)} \quad\left(z \in \Omega, u \in P_{z}\right)
$$

Consider a $C^{1}$ path $\eta:[0,1] \rightarrow P_{z}$ from $\eta(0)=z$ to $\eta(1)=w$; let $g(t)=f(\eta(t))$. Then $g$ satisfies the differential equation

$$
\begin{aligned}
g^{\prime}(t) & =\sum_{j=1}^{s} \beta_{j}\left(\eta^{\prime}(t), \eta(t)\right)(g(t))^{(2 j+1)}, \\
g(0) & =f(z)
\end{aligned}
$$


Let

$$
f(z)=\sum_{k=1}^{s} \alpha_{k} \varepsilon_{k}
$$

be the spectral decomposition of $f(z)$. Let $h:[0,1] \rightarrow \mathbb{C}^{s}$ be the solution of the differential system

$$
\begin{aligned}
h_{k}^{\prime}(t) & =\sum_{j=1}^{s} \beta_{j}\left(\eta^{\prime}(t), \eta(t)\right)\left(h_{k}(t)\right)^{2 j+1)} \quad(1 \leq k \leq s), \\
h_{k}(0) & =\alpha_{k} .
\end{aligned}
$$

Then the solution of (4.10) is

$$
g(t)=\sum_{k=1}^{s} h_{k}(t) \varepsilon_{k}
$$

which shows that $g(1)=f(w)$ belongs to $P_{f(z)}$.

Let $K$ denote the group of linear automorphisms of $\Omega$.

Proposition 5. Let $f: \Omega \rightarrow \Omega$ be a diffeomorphism of $\Omega$ such that

$$
B(f(z), f(z)) \circ \mathrm{d} f(z)=\mathrm{d} f(z) \circ B(z, z)
$$

for all $z \in \Omega$. Then any $K$-orbit is globally invariant by $f$.

Proof. Assume first that $z=\lambda_{1} e_{1}+\cdots+\lambda_{r} e_{r}$ is super regular. Then $f(z)=$ $\mu_{1} \varepsilon_{1}+\cdots+\mu_{r} \varepsilon_{r}$, and equality between the eigenvalues of $B(z, z)$ and $B(f(z), f(z))$ implies $\lambda_{j}=\mu_{j}$, hence $f(z) \in K z$ and $f(K z)=K z$. By continuity, this also holds for any $z \in \Omega$.

4.3. We now go back to bisysmplectomorphisms of $\Omega$.

Proposition 6. Let $\Omega$ be a bounded circled symmetric domain and denote by $K$ the group of linear automorphisms of $\Omega$. For each bisymplectomorphism $f \in \mathcal{B}(\Omega)$, we have $\mathrm{d} f(0) \in K$.

As $K \subset \mathcal{B}(\Omega)$, it will be sufficient to study the subgroup

$$
\mathcal{B}_{0}(\Omega)=\left\{f \in \mathcal{B}(\Omega) \mid \mathrm{d} f(0)=\mathrm{id}_{V}\right\} .
$$

Proof. Let $\left(e_{1}, \ldots, e_{r}\right)$ be a frame of $V$ and let $V=\bigoplus V_{i j}$ be the associated simultaneous Peirce decomposition. Consider a regular element

$$
z=\alpha_{1} e_{1}+\cdots+\alpha_{r} e_{r},
$$

$1>\alpha_{1}>\cdots>\alpha_{r}>0$. For $f \in \mathcal{B}(\Omega)$ and $z$ super-regular, we have $f(z)=\alpha_{1} \varepsilon_{1}+$ $\cdots+\alpha_{r} \varepsilon_{r}$, where $\left(\varepsilon_{1}, \ldots, \varepsilon_{r}\right)$ is a frame of $V$, which may depend on $\left(\alpha_{1}, \ldots, \alpha_{r}\right)$. As $V_{j j}=\mathbb{C} e_{j}$ is the eigenspace of $B(z, z)$ for the eigenvalue $\left(1-\alpha_{j}^{2}\right)^{2}$, we deduce from (4.4) that

$$
\mathrm{d} f(z)\left(e_{j}\right) \in \mathbb{C} \varepsilon_{j} .
$$

By continuity, there exists a frame $\left(e_{1}^{\prime}, \ldots, e_{r}^{\prime}\right)$ such that

$$
\mathrm{d} f(0)\left(e_{j}\right) \in \mathbb{C} e_{j}^{\prime} \quad(1 \leq j \leq r) .
$$


Multiplying $e_{j}^{\prime}$ by a suitable complex of modulus 1 , we may assume that

$$
\mathrm{d} f(0)\left(e_{j}\right)=\lambda_{j}^{\prime} e_{j}^{\prime}, \quad \lambda_{j}^{\prime}>0 \quad(1 \leq j \leq r) .
$$

We have also

$$
\mathrm{d} f(0)\left(e_{j}\right)=\lim _{t \rightarrow 0+} \frac{f\left(t e_{j}\right)}{t}
$$

and $f\left(t e_{j}\right)$ is a multiple of a minimal tripotent, with spectral norm $\left\|f\left(t e_{j}\right)\right\| \leq$ $t\left\|e_{j}\right\|$, hence $\left\|\mathrm{d} f(0)\left(e_{j}\right)\right\| \leq\left\|e_{j}\right\|$ and $\lambda_{j}^{\prime} \leq 1$. The same argument applied to $f^{-1}$ gives $\lambda_{j}^{\prime}=1$. So the image of a frame $\left(e_{1}, \ldots, e_{r}\right)$ under $\mathrm{d} f(0)$ is a frame $\left(e_{1}^{\prime}, \ldots, e_{r}^{\prime}\right)$ and the Peirce spaces $V_{j k}$ relative to $\left(e_{1}, \ldots, e_{r}\right)$ are mapped by $\mathrm{d} f(0)$ onto the corresponding Peirce spaces $V_{j k}^{\prime}$ relative to $\left(e_{1}^{\prime}, \ldots, e_{r}^{\prime}\right)$. In particular, $\mathrm{d} f(0)$ is an $\mathbb{R}$-linear map from $\mathbb{C} e_{j}$ onto $\mathbb{C} e_{j}^{\prime}$. We have $\mathrm{d} f(0)\left(\mathrm{i} e_{j}\right)=\beta e_{j}^{\prime}$, with $|\beta|=1$. As

$$
\omega_{0}(u, v)=\frac{\mathrm{i}}{2}(\operatorname{tr} D(u, v)-\operatorname{tr} D(v, u)),
$$

we have

$$
\begin{aligned}
\omega_{0}\left(e_{j}, \mathrm{i} e_{j}\right) & =\operatorname{tr} D\left(e_{j}, e_{j}\right), \\
\omega_{0}\left(\mathrm{~d} f(0)\left(e_{j}\right), \mathrm{d} f(0)\left(\mathrm{i} e_{j}\right)\right) & =\operatorname{Im} \beta \operatorname{tr} D\left(e_{j}^{\prime}, e_{j}^{\prime}\right) .
\end{aligned}
$$

From $\operatorname{tr} D\left(e_{j}^{\prime}, e_{j}^{\prime}\right)=\operatorname{tr} D\left(e_{j}, e_{j}\right)$ and $f^{*} \omega_{0}=\omega_{0}$, we get $\operatorname{Im} \beta=1, \beta=\mathrm{i}$, which means that $\mathrm{d} f(0)$ is $\mathbb{C}$-linear on $\mathbb{C} e_{1} \oplus \cdots \oplus \mathbb{C} e_{r}$. Finally, $\mathrm{d} f(0)$ is $\mathbb{C}$-linear on $V$, maps minimal tripotents to minimal tripotents and $\Omega$ to $\Omega$.

4.4. The unit disc. Let $\Delta$ be the unit disc of $\mathbb{C}$. The associated triple product is $\{u, v, w\}=2 u \bar{v} w$. The Bergman operator is $B(z, z) w=\left(1-|z|^{2}\right)^{2} w$. The Bergman metric is

$$
h_{z}(u, v)=\frac{u \bar{v}}{\left(1-|z|^{2}\right)^{2}} .
$$

The two symplectic forms are

$$
\begin{aligned}
& \omega_{0}=\frac{\mathrm{i}}{2} \mathrm{~d} z \wedge \mathrm{d} \bar{z}, \\
& \omega_{-}=\frac{\omega_{0}}{\left(1-|z|^{2}\right)^{2}} .
\end{aligned}
$$

Denote by $S^{1}$ the unit circle in $\mathbb{C}$ and consider the "polar coordinates" diffeomorphism

$$
\begin{aligned}
\Theta:(0,1) \times S^{1} & \rightarrow \Delta \backslash\{0\}, \\
(r, \zeta) & \mapsto r \zeta .
\end{aligned}
$$

Then we have

$$
\Theta^{*} \omega_{0}=r \mathrm{~d} r \wedge \frac{\mathrm{d} \zeta}{\mathrm{i} \zeta}
$$

The following theorem characterizes the elements of $\mathcal{B}(\Delta)$.

Theorem 2. The elements $f \in \mathcal{B}(\Delta)$ are the maps defined by

$$
f(z)=u\left(|z|^{2}\right) z \quad(z \in \Delta),
$$

where $u$ is a smooth function $u:[0,1) \rightarrow S^{1} \simeq U(1)$. 
In other words, the restriction of $f$ to a circle of radius $r(0<r<1)$ is the rotation $u\left(r^{2}\right)$.

Proof. From (4.14), we see that a diffeomorphism $f: \Delta \rightarrow \Delta$ is a bisymplectomorphism if and only if $f$ preserves $\omega_{0}$ and $|f(z)|=|z|$ for all $z \in \Delta$.

If $|f(z)|=|z|$ for all $z \in \Delta$, the map $F=\Theta^{-1} \circ f \circ \Theta$ may be written

$$
(r, \zeta) \stackrel{F}{\rightarrow}(r, Z(r, \zeta))
$$

for some smooth function $Z:(0,1) \times S^{1} \rightarrow S^{1}$. We have

$$
F^{*}\left(\Theta^{*} \omega_{0}\right)=r \mathrm{~d} r \wedge \frac{\mathrm{d} Z}{\mathrm{i} Z}=r \mathrm{~d} r \wedge \frac{\mathrm{d}_{\zeta} Z}{\mathrm{i} Z} .
$$

If $f$ preserves $\omega_{0}$, then $F$ preserves $\Theta^{*} \omega_{0}$, which implies

$$
\frac{\mathrm{d}_{\zeta} Z}{Z}=\frac{\mathrm{d} \zeta}{\zeta} .
$$

For $r$ fixed, let $u_{r}(\zeta)=Z(r, \zeta)$; the condition (4.15) is then equivalent to

$$
\frac{\mathrm{d} u_{r}}{u_{r}}=\frac{\mathrm{d} \zeta}{\zeta}
$$

for all $r \in(0,1)$. The condition (4.16) is in turn equivalent to

$$
u_{r}(\zeta)=v(r) \zeta
$$

with $v(r) \in S^{1}$. The function $v$, which is given by

$$
v(r)=\frac{Z(r, \zeta)}{\zeta}
$$

is smooth on $(0,1)$ and

$$
f(r \zeta)=r v(r) \zeta
$$

or

$$
f(z)=v(|z|) z
$$

for $z \in \Delta, z \neq 0$; on the other hand, $f(0)=0$. Let $g$ be the restriction of $f$ to $(-1,1)$; from (4.18), we see that $g$ is odd. For $x \neq 0$, we have

$$
g(x)=\int_{0}^{1} \frac{\mathrm{d}}{\mathrm{d} s} g(s x) \mathrm{d} s=x \int_{0}^{1} g^{\prime}(s x) \mathrm{d} s,
$$

which shows that

$$
v(r)=\int_{0}^{1} g^{\prime}(s r) \mathrm{d} s
$$

extends to a smooth even function $v:(-1,1) \rightarrow S^{1}$. Let $u:[0,1) \rightarrow S^{1}$ be defined by

$$
u(r)=v(\sqrt{r}) .
$$

It follows then from Whitney's theorem [5] that $u$ is smooth at 0 .

Conversely, if

$$
f(z)=u\left(|z|^{2}\right) z,
$$

with $u:[0,1) \rightarrow S^{1}$ smooth, then $f$ satisfies $|f(z)|=|z|, f$ is a diffeomorphism with inverse $f^{-1}(w)=u\left(|w|^{2}\right)^{-1} w$; also, $f$ preserves $\omega_{0}$ on $\Delta \backslash\{0\}$, hence on $\Delta$ by continuity. This implies that $f \in \mathcal{B}(\Delta)$. 
4.5. The polydisc. Let $\Delta^{r} \subset \mathbb{C}^{r}$ be the product of $r$ unit discs. The Jordan triple product on $V=\mathbb{C}^{r}$ is just the component-wise product

$$
\{x, y, z\}=2\left(x_{1} \overline{y_{1}} z_{1}, \ldots, x_{r} \overline{y_{r}} z_{r}\right) .
$$

Let $\left(e_{1}, \ldots, e_{r}\right)$ denote the canonical basis of $\mathbb{C}^{r}$. The minimal tripotents of $V$ are the elements $\lambda_{j} e_{j}, 1 \leq j \leq r,\left|\lambda_{j}\right|=1$. Any frame (maximal ordered set of mutually orthogonal tripotents) has the form

$$
\left(\lambda_{j} e_{\sigma(j)}\right)_{1 \leq j \leq r},
$$

where $\left|\lambda_{j}\right|=1$ and $\sigma \in \mathfrak{S}_{r}$ is a permutation of $\{1, \cdots, r\}$. The corresponding Peirce decomposition is $V=\mathbb{C} e_{\sigma(1)} \oplus \cdots \oplus \mathbb{C} e_{\sigma(r)}$.

Theorem 3. A diffeomorphism $f: \Delta^{r} \rightarrow \Delta^{r}$ belongs to $\mathcal{B}_{0}\left(\Delta^{r}\right)$ if and only if there exist smooth functions $u_{j}:[0,1) \rightarrow S^{1}$ such that $u_{j}(0)=1$ and

$$
f\left(z_{1}, \ldots, z_{r}\right)=\sum_{j=1}^{r} u_{j}\left(\left|z_{j}\right|^{2}\right) z_{j} e_{j} \quad\left(z_{j} \in \Delta\right) .
$$

Proof. Let $f \in \mathcal{B}_{0}\left(\Delta^{r}\right)$ be a bisymplectomorphism with $\mathrm{d} f(0)=\mathrm{id}_{V}$. Consider a regular element $z \in \Delta^{r}$, that is,

$$
z=z_{1} e_{1}+\cdots+z_{r} e_{r},
$$

with all $\left|z_{j}\right|$ different. The spaces $\mathbb{C} e_{j}$ of the corresponding Peirce decomposition are mapped by $\mathrm{d} f(z)$ to the spaces $\mathbb{C} e_{\sigma(j)}$ of another Peirce decomposition, for some permutation $\sigma \in \mathfrak{S}_{r}$. This means that $\left[\mathrm{d} f(z)\left(e_{j}\right)\right]=\left[e_{\sigma(j)}\right]$ for all regular $z \in \Delta^{r}$, where [ ] denotes the class in $\mathbb{P}\left(\mathbb{C}^{r}\right)$; by continuity, this is true for all $z \in \Delta^{r}$. As $\left\{\left[e_{1}\right], \ldots,\left[e_{r}\right]\right\}$ is discrete in $\mathbb{P}\left(\mathbb{C}^{r}\right)$ and $\mathrm{d} f(0)=\mathrm{id}$, we have $\left[\mathrm{d} f(z)\left(e_{j}\right)\right]=\left[e_{j}\right]$ and

$$
\mathrm{d} f(z)\left(\mathbb{C} e_{j}\right)=\mathbb{C} e_{j}
$$

for all $z \in \Delta^{r}$. This shows that $f\left(z_{1}, \ldots, z_{r}\right)=\sum_{j=1}^{r} f_{j}(z) e_{j}$, with $\left|f_{j}(z)\right|=\left|z_{j}\right|$. From (4.20), we deduce that $f_{j}$ depends only of $z_{j}$. Each $f_{j}$ is then a bisymplectomorphism of the unit disc. According to Theorem 2 there exists a smooth function $u_{j}:[0,1) \rightarrow S^{1} \simeq U(1)$ such that

$$
f_{j}\left(z_{j}\right)=u_{j}\left(\left|z_{j}\right|^{2}\right) z_{j} \quad\left(z_{j} \in \Delta\right) .
$$

Finally, any $f \in \mathcal{B}_{0}\left(\Delta^{r}\right)$ has the form (4.19). Conversely, each $f$ of the form (4.19) is easily seen to be a bisymplectomorphism.

4.6. The general case. We assume now that the domain $\Omega$ is irreducible, that is, not a product of two bounded symmetric domains. In Theorem 4, we will characterize the bisymplectomorphisms of $\Omega$. This theorem may be considered as a kind of Schwarz lemma.

Let $f \in \mathcal{B}_{0}(\Omega)$. If $z=\sum \lambda_{j} e_{j}$ is a regular element, then $P_{z}$ is the poly$\operatorname{disc}\left(\mathbb{C} e_{1} \oplus \cdots \oplus \mathbb{C} e_{r}\right) \cap \Omega$ and $f\left(P_{z}\right)=P_{z}$. For a frame $\mathbf{e}=\left(e_{1}, \ldots, e_{r}\right)$, let $P(\mathbf{e})=\left(\mathbb{C} e_{1} \oplus \cdots \oplus \mathbb{C} e_{r}\right) \cap \Omega$. By the same argument as in the proof of Theorem 3, the restriction of $f$ to $P(\mathbf{e})$ has the form

$$
\sum_{j=1}^{r} \lambda_{j} e_{j} \mapsto \sum \lambda_{j} u_{j}\left(\left|\lambda_{j}\right|^{2}\right) e_{j}
$$


where the $u_{j}$ 's are smooth functions $u_{j}:[0,1) \rightarrow S^{1} \simeq U(1)$. In the "polar coordinates" $\left(\left(\lambda_{1}, \ldots, \lambda_{r}\right), \mathbf{e}=\left(e_{1}, \ldots, e_{r}\right)\right)$, the map $f$ is then represented by

$$
\left(\left(\lambda_{1}, \ldots, \lambda_{r}\right), \mathbf{e}\right) \mapsto\left(\left(\lambda_{1}, \ldots, \lambda_{r}\right),\left(u_{1}\left(\lambda_{1}^{2}, \mathbf{e}\right) e_{1}, \ldots, u_{r}\left(\lambda_{r}^{2}, \mathbf{e}\right) e_{r}\right)\right) .
$$

Let $w_{j}\left(\lambda_{j}, \mathbf{e}\right)=u_{j}\left(\lambda_{j}^{2}, \mathbf{e}\right)$. We obtain

$$
\begin{aligned}
f^{*} \eta_{j j} & =\frac{\mathrm{i}}{2} f^{*} \sum_{m=1}^{n} e_{j m} \mathrm{~d} \bar{e}_{j m}=\frac{\mathrm{i}}{2} \sum_{m=1}^{n} w_{j} e_{j m}\left(\bar{w}_{j} \mathrm{~d} \bar{e}_{j m}+\bar{e}_{j m} \mathrm{~d} \bar{w}_{j}\right) \\
& =\eta_{j j}-\frac{\mathrm{i}}{2}\left(e_{j} \mid e_{j}\right) \frac{\mathrm{d} w_{j}}{w_{j}} \\
f^{*} \omega_{j j} & =\omega_{j j} .
\end{aligned}
$$

As $\Omega$ is irreducible, $\left(e_{j} \mid e_{j}\right)$ has the same value $g$ for all minimal tripotents. Finally

$$
\begin{aligned}
f^{*} \omega_{0} & =\sum_{j=1}^{r} \lambda_{j}^{2} \omega_{j j}+\sum_{j=1}^{r} \lambda_{j} \mathrm{~d} \lambda_{j} \wedge\left(\eta_{j j}-\mathrm{i} g \frac{\mathrm{d} w_{j}}{w_{j}}\right) \\
& =\omega_{0}-\mathrm{i} g \sum_{j=1}^{r} \lambda_{j} \mathrm{~d} \lambda_{j} \wedge \frac{\mathrm{d} w_{j}}{w_{j}} .
\end{aligned}
$$

Then $f^{*} \omega_{0}=\omega_{0}$ implies that $f \in \mathcal{B}_{0}(\Omega)$, written in the form (4.21), satisfies

$$
\sum_{j=1}^{r} \lambda_{j} \mathrm{~d} \lambda_{j} \wedge \frac{\mathrm{d} w_{j}}{w_{j}}=0
$$

As $w_{j}$ depends only on $\lambda_{j}$ and $\mathbf{e}$, this implies that $\mathrm{d}_{\mathbf{e}} w_{j}=0$. As the manifold of frames is connected when the domain $\Omega$ is irreducible, $w_{j}$ does not depend on $\mathbf{e} \in \mathcal{F}$. As a permutation of a frame is again a frame, we have $w_{1}=\cdots=w_{r}$ and $u_{1}=\cdots=u_{r}$.

Finally, an element $f \in \mathcal{B}_{0}(\Omega)$ is written in polar coordinates

$$
\left(\left(\lambda_{1}, \ldots, \lambda_{r}\right),\left(e_{1}, \ldots, e_{r}\right)\right) \mapsto\left(\left(\lambda_{1}, \ldots, \lambda_{r}\right),\left(u\left(\lambda_{1}^{2}\right) e_{1}, \ldots, u\left(\lambda_{r}^{2}\right) e_{r}\right)\right),
$$

where $u$ is a smooth function $u:[0,1) \rightarrow S^{1} \simeq U(1)$.

Theorem 4. Let $\Omega$ be an irreducible Hermitian bounded circled symmetric domain and let $K$ be the isotropy group of 0 . The analytic (resp. $C^{\infty}$ ) bisymplectomorphisms of $\Omega$ are the maps $\phi=f \circ g$, where $g=\mathrm{d} \phi(0) \in K$ and $f$ is associated to $v(t)=t u\left(t^{2}\right)$, with $u:[0,1) \rightarrow S^{1} \simeq U(1)$ analytic (resp. $\left.C^{\infty}\right)$ and $u(0)=1$.

\section{REFERENCES}

[1] Di Scala, A.J. and Loi, A., Symplectic duality of symmetric spaces, Advances in Math., 217 (2008), 2336-2352.

[2] Loos, Ottmar, Bounded symmetric domains and Jordan pairs, Math. Lectures, Univ. of California, Irvine, 1977.

[3] McDuff, Dusa, The symplectic structure of Kähler manifolds of non-positive curvature, J. Differ. Geom., 28(3), (1988), 467-475.

[4] Roos, Guy, Jordan triple systems, pp. 425-534, in J. Faraut, S. Kaneyuki, A. Korányi, Q.k. Lu, G. Roos, Analysis and geometry on complex homogeneous domains, Progress in Mathematics, vol. 185, Birkhäuser, Boston, 2000.

[5] Whitney, H., Differentiable even functions, Duke Math. J., 10 (1943), 159-160. 
A.D.: Dipartimento di Matematica, Politecnico di Torino, Corso Duca degli Abruzzi 24, 10129 TORINO, ITALY

E-mail address: antonio.discala@polito.it

A.L.: Dipartimento di Matematica e Informatica, Università di Cagliari, Via OsPEdale 72, 09124 Cagliari, Italy

E-mail address: loi@unica.it

G.R.: Nevski prospekt 113/4-53, 191024 St Petersburg, Russian Federation

E-mail address: guy.roos@normalesup.org 